\title{
Sequencing Time Window for the Total Product Rate Variation Problem
}

\author{
Shree Ram Khadka \\ Central Department Mathematics, Institute of Science and Tchnology \\ Tribhuvan University, Kathmandu, Nepal \\ E-mail: shreeramkhadka@gmail.com \\ P.O.Box, 13143
}

\begin{abstract}
The problem of minimizing the total deviations between the actual and the ideal cumulative production of a variety of models of a common base product arises as a sequencing problem in mixed-model just-in-time production systems. This is called the total product rate variation problem. Several pseudo-polynomial exact algorithms and heuristics have been derived for this problem. In this paper, we estimate the sequencing time window for all copies of all models of a common base product for the problem when the m-th power of the total deviations between the actual and the ideal cumulative productions has to be minimized.
\end{abstract}

Key words: Bound, product rate variation problem, non-linear integer programming problem.

\section{INTRODUCTION}

Many companies have changed the assembly lines from paced single-model lines for mass production to mixedmodel assembly lines for mass customization of a variety of models of a common base product. Just-intime production system which requires producing only the necessary products in the necessary quantities at the necessary times usually uses mixed-model assembly lines.

Mixed-model just-in-time production systems with negligible change-over costs between the models have been used in order to respond to the customer demands for a variety of models of a common base product without holding large inventories or incurring large shortages. One of the most important problems for the effective utilization of the systems consists in sequencing different models with keeping the rate of usage of all parts used by the assembly lines as constant as possible. The problem is known as the mixed-model just-in-time sequencing problem (abbreviated as MMJITSP). The problem of minimizing the variation in the rate at which different models are produced on the line is called the product rate variation problem (abbreviated as PRVP). The latter problem is the singlelevel case of MMJITSP. The problem of minimizing the total deviations between the actual cumulative productions from the ideal one is called the total PRVP (abbreviated as TPRVP), see Kubiak (1993). This problem has been widely investigated in the literature since it has a model with a strong mathematical base and wide real-world applications, see Dhamala \& Khadka (2009), a recent survey.
In this paper, we determine the sequencing time window for all copies of all models of a common base product. The remainder of the paper is as follows. In the second section, we present a mathematical modeling of the problem. In the third section, we present the sequencing time window for all copies of all models of a common base product which is the major contribution of this paper.

First, the level curves are investigated, then the largest function value and finally the smallest function value. The last section concludes the paper.

\section{MATHEMATICAL MODELLING}

Let $D$ be the total demand of $\mathrm{n}$ different models with $d_{i}$ copies of model $i, i=1,2, \ldots, n$, where $n \geq 2$ and $D=\sum_{i=1}^{n} d_{i}$. The time horizon is partitioned into $D$ equal time units under the assumption that each copy of a model $i, i=1, \ldots, n$, has equal processing time. A copy of a model is produced in a time unit $k, k=$ $1, \ldots, D$, means that the copy of the model is produced during the time period from $k-1$ to $k$. Let $r_{i}=\frac{d_{i}}{D}$ be the demand rate. Let $x_{i k}$ and $k r_{i}$ be the actual and the ideal cumulative productions, respectively, of model $i$ produced during the time units 1 through $k$. An inventory holds if $x_{i k}-k r_{i}>0$, and a shortage incurs if $k r_{i}-x_{i k}>0$. We assign the same cost for both inventory and shortage. Miltenburg (1989) and Kubiak and Sethi $(1991,1994)$ gave an integer programming formulation for TPRVP as follows

with $m$ being a positive integer:

$\operatorname{minimize}\left[F_{m}=\sum_{k=1}^{D} \sum_{i=1}^{n}\left|x_{i k}-k r_{i}\right|^{m}\right]$ 
subject to

$$
\begin{aligned}
& \sum_{i=1}^{n} x_{i k}=k, \quad k=1,2, \ldots, D \\
& x_{i(k-1)} \leq x_{\mathrm{ik}}, \quad i=1,2, \ldots, n ; k=2,3, \ldots, D \\
& x_{i D}=d_{i}, x_{i o}=0, \quad i=1,2, \ldots, n \\
& x_{i k} \geq 0 \text {, integer } i=1,2, \ldots, n ; k=1,2, \ldots, D \text {. }
\end{aligned}
$$

\section{LEVEL CURVE AND BOUNDS}

There exist $n D$ deviations between the actual and the ideal cumulative productions of $D$ copies of $n$ models. The value of the actual cumulative production $x_{i k}$, $i=1,2, \ldots, n ; k=1,2, \ldots, D$, is sequence-dependent integer from $\left\{0,1, \ldots, d_{i}\right\}$. However, the value of the ideal cumulative production $k r_{i}, i=1,2, \ldots, n ; k=$ $1,2, \ldots, D$, is sequence-independent rational number. Let $j$ be the number of copies of a model and $(i, j)$ be the $j^{\text {th }}$ copy of model $i, i=1,2, \ldots, n$. The actual cumulative production $x_{i k}, i=1,2, \ldots, n ; k=$ $1,2, \ldots, D$, has $n D$ values with $x_{i k} \in\{j \mid j=$ $\left.0,1,2, \ldots, d_{i} ; i=1,2, \ldots, n\right\}$. There exist at most $n+D$ different values of $x_{i k}$ for TPRVP. Hence, one can replace $x_{i k}$ by $j$ with $j=0,1, \ldots, d_{i} ; i=$ $1,2, \ldots, n$, in the level curve of the objective value of the function of TPRVP. The level curve for copy $(i, j)$ of the objective function of TPRVP is defined as

$f_{i j}{ }^{m}=n D\left|j-k r_{i}\right|^{m}, i=1,2, \ldots, n ; j=0,1, \ldots, d i$.

A perfect matching of copies and time units relies on the level curves and the bound $B>0$ of the function $F_{m}$ of PRVP that are drawn over the planning horizon. The points at which the bound intersects the level curves are useful to find the sequencing times. A copy $(i, j)$ is sequenced at a time unit $k \in\{1, \ldots, D\}$ such that the level curve does not exceed the bound $B$. An upper bound on the absolute deviation objective function Ixik - kril for BPRVP is $U B^{*}{ }_{1}=1$, see Steiner and Yeomans (1993), and a better one has been given as $U B^{*}{ }_{1}=1-$ $\frac{1}{D}$, see Brauner and Crama (2004). Since the points, where the bound $U B^{*}{ }_{1}=1$ intersects the level curves of BPRVP with the objective function $\left|x_{i k}-k r_{i}\right|^{m}$ for different values of $m$, are the same, the bound $U B^{*}{ }_{1}=1$ is also an upper bound for BPRVP with the objective function $\left|x_{i k}-k r_{i}\right|^{m}$ for all values of $m$. However, the upper bounds corresponding to $U B^{*}{ }_{1}=1-\frac{1}{D}$ are different for BPRVP with the objective functions $\left|x_{i k}-k r_{i}\right|^{m}$ for different values of $m$. An upper bound on the largest value of the objective function $\mid x_{i k}-$ $\left.k r_{i}\right|^{m}$ of BPRVP has been established as

$$
U B^{*}{ }_{m}=\left(1-\frac{1}{D}\right)^{m}
$$

Dhamala et al. (2010) and Khadka (2012). A minor modification yields an upper bound on the optimal value of function $F_{m}$ for PRVP given by

$U B_{m}=n D\left(1-\frac{1}{D}\right)^{m}$, Khadka (2014) (although it not tight). The lower bound $L B^{*}{ }_{1}=1-r_{\max }$ on the absolute deviation objective function $\left|x_{i k}-k r_{i}\right|$ for BPRVP has been established by Steiner and Yeomans (1993), and it has been modified as

$L B^{*}{ }_{m}=\left(1-r_{\max }\right)^{m}$, for this problem with the objective function $\left|x_{i k}-k r_{i}\right|^{m}$ (Dhamala et al. (2010) and Khadka (2012)). Hence, a lower bound on the optimal value of the objective function $F_{m}$ for TPRVP is given by

$$
L B_{m}=n D\left(1-r_{\max }\right)^{m} \text {, Khadka (2014). }
$$

\section{SEQUENCING TIME WINDOW}

The earliest and the latest sequencing times are determined by the level curve and a suitably chosen bound. The selection of an upper bound always yields the sequencing times that give rise to a feasible solution. However, this is time-consuming. A selection of a lower bound for small-size instances is better for BPRVP (see Kovalyov et al. (2001)). A feasible solution corresponding to the lower bound is optimal.

Let $E_{m}(i, j)$ and $L_{m}(i, j)$, be the earliest and the latest sequencing times of copy $(i, j)$ for a given (suitably chosen) bound $B$, respectively, when considering the objective function $F_{m}$.

The integer time interval $T_{(i, j) m}=\left[E_{m}(i, j), L_{m}(i, j)\right]$

is called the sequencing time window for the copy $(i, j)$ since the level curve of the objective function $F_{m}$ of TPRVP does not exceed the bound B if copy $(i, j)$ is sequenced within this window. The earliest sequencing time $E_{m}(i, j) \in\{1, \ldots, D\}$ is the unique integer such that, when copy $(i, j)$ is sequenced at time $E_{m}(i, j)-1$, the level curve exceeds the bound $B$ but does not exceed this bound when sequenced at $E_{m}(i, j)$.

Similarly, the latest sequencing time $L_{m}(i, j) \in$ $\{1, \ldots, D\}$ is the unique integer such that, when copy $(i,(j-1))$ is sequenced at time $L_{m}(i, j)-1$, the level curve does not exceed the bound $B$ but it exceeds this bound when sequenced at time $L_{m}(i, j)$.

Theorem 1. Let $E_{m}(i, j)$ and $L_{m}(i, j), i=1, \ldots, n ; j=$ $0,1, \ldots, d_{i}$, be the earliest and the latest sequencing times, respectively, for a given (suitably chosen) bound $B>0$ for an instance $\left(d_{1}, \ldots, d_{n}\right)$. Then the equalities $E_{m}(i, j)=\left\lceil\frac{j-\sqrt[m]{\frac{B}{n D}}}{r_{i}}\right\rceil$ and $L_{m}(i, j)=\left\lfloor\frac{j-1+\sqrt[m]{\frac{B}{N D}}}{r_{i}}+1\right\rfloor$ hold. 
Proof. Let $\operatorname{Em}(i, j)$ and $\operatorname{Lm}(i, j), i=1, \ldots, n ; j=$ $0,1, \ldots, d_{i}$, be the earliest and the latest sequencing times, respectively.

The time $\operatorname{Em}(i, j)$ satisfies the two inequalities

$$
\begin{array}{r}
n D\left[j-\left(E_{m}(i, j)-1\right) r_{i}\right]^{m}>B \text { and } \\
n D\left[j-E_{m}(i, j) r_{i}\right]^{m} \leq B
\end{array}
$$

This implies the inequalities

$$
1+\frac{j-\sqrt[m]{\frac{B}{n D}}}{r_{i}}>\operatorname{Em}(i, j) \text { and } \frac{j-\sqrt[m]{\frac{B}{n D}}}{r_{i}} \leq \operatorname{Em}(i, j)
$$

Therefore, we have the inequalities

$\frac{j-\sqrt[m]{\frac{B}{n D}}}{r_{i}} \leq E m(i, j)<1+\frac{j-\sqrt[m]{\frac{B}{n D}}}{r_{i}}$

Hence, we obtain $E_{m}(i, j)=\left\lceil\frac{j-\sqrt[m]{\frac{B}{N D}}}{r_{i}}\right\rceil$

Similarly, the time $\operatorname{Lm}(i, j)$ satisfies the inequalities

$$
\begin{array}{r}
n D\left[\left(L_{m}(i, j)-1\right) r i-(j-1)\right]^{m} \leq B \text { and } \\
n D\left[L_{m}(i, j) r i-(j-1)\right]^{m}>B
\end{array}
$$

This implies the inequalities

$$
1+\frac{j-1+\sqrt[m]{\frac{B}{n D}}}{r_{i}} \geq \operatorname{Lm}(i, j) \text { and } \frac{j-1+\sqrt[m]{\frac{B}{n D}}}{r_{i}}<\operatorname{Lm}(i, j)
$$

We have $\frac{j-1+\sqrt[m]{\frac{B}{n D}}}{r_{i}}<\operatorname{Lm}(i, j) \leq 1+\frac{j-1+\sqrt[m]{\frac{B}{n D}}}{r_{i}}$

Thus, we obtain $\quad L_{m}(i, j)=\left\lfloor\frac{j-1+\sqrt[m]{\frac{B}{N D}}}{r_{i}}+1\right\rfloor$

Hence the result.

Corollary 1 Let $E_{m}(i, j)$ and $L_{m}(i, j), i=1, \ldots, n ; j=$ $1, \ldots, d_{i}$, be the earliest and the latest sequencing times, respectively. Then the inequality $E_{m}(i, j) \leq L_{m}(i, j)$ holds.

Proof. Consider the earliest sequencing time

$E_{m}(i, j)=\left\lceil\frac{j-\sqrt[m]{\frac{B}{n D}}}{r_{i}}\right\rceil$

Without loss of optimality, one can use the bound $B=U B_{m}$, i.e., $B=n D\left(1-\frac{1}{D}\right)^{m}$.

Thus, we obtain

$$
\begin{array}{r}
E_{m}(i, j)=\left\lceil\frac{j-\left(1-\frac{1}{D}\right)}{r_{i}}\right\rceil=\left\lceil\frac{j-1+\left(1-\frac{1}{D}\right)}{r_{i}}+\frac{1+2\left(\frac{1}{D}-1\right)}{r_{i}}\right\rceil \\
\left.\leq \mid \frac{j-1+\left(1-\frac{1}{D}\right)}{r_{i}}+1+\frac{1+2\left(\frac{1}{D}-1\right)}{r_{i}}\right\rceil
\end{array}
$$

Since $\frac{1+2\left(\frac{1}{D}-1\right)}{r_{i}} \leq o$ for any $D \geq 2$, we can write

$$
E_{m}(i, j) \leq\left\lfloor\frac{j-1+\left(1-\frac{1}{D}\right)}{r_{i}}+1\right\rfloor=\left\lfloor\frac{j-1+\sqrt[m]{\frac{B}{N D}}}{r_{i}}+1\right\rfloor=L_{m}(i, j)
$$

Corollary 2 Let $[1, D]$ be the planning horizon and

$$
T_{(i, j) m}, i=1, \ldots, n ; j=1, \ldots, d_{i},
$$

be the sequencing time window for copy $(i, j)$. Then the inclusion $T_{(i, j) m} \subseteq[1, D]$

holds for $i=1, \ldots, n ; j=1, \ldots, d_{i}$.

Proof. Suppose that copy $(i, j), i=1, \ldots, n ; j$

$=1, \ldots, d_{i}$, is sequenced at time $k \in T_{(i, j) m}$.

Thus, $E_{m}(i, j) \leq k \leq L_{m}(i, j)$.

The minimum value of the earliest sequencing time $E_{m}(i, j)$ for a copy $(i, j)$ can be the first time unit 1 . Likewise, the maximum value of the latest sequencing time $L_{m}(i, j)$ for a copy $(i, j)$ can be the last time unit $D$. i.e., $1 \leq E_{m}(i, j)$ and $L_{m}(i, j) \leq D$.

Thus, $1 \leq k \leq D$.

Hence, the inclusion $\quad T_{(i, j) m} \subseteq[1, D]$ holds.

Corollary 3 Let $[1, D]$ be the planning horizon and

$T_{(i, j) m}, i=1, \ldots, n ; j=1, \ldots, d_{i}$,

be the sequencing time window for copy $(i, j)$. Then

$\cup^{n . d_{i}}{ }_{i, j=1} T_{(i, j) m}=[1, D]$.

Proof. Since, by using Corollary $2, T_{(i, j) m} \subseteq[1, D]$

for each copy $(i, j), i=1, \ldots, n ; j=1, \ldots, d_{i}$, we have $\cup^{n \cdot d_{i}}{ }_{i, j=1} T_{(i, j) m} \subseteq[1, D]$

The planning horizon is partitioned into exactly $D$ equal time units, where $D$ is equal to the total number of all copies $j, j=1, \ldots, d_{i}$, of all models $i, i=1, \ldots, n$. There exists at least one sequencing time window that contains the first time unit 1 and at least another one that contains the last time unit $D$. This implies $[1, D] \subseteq$ $\cup^{n . d_{i}}{ }_{i, j=1} T_{(i, j) m}$

Corollary 4 Given a suitably chosen bound $B>0$, the level curve of the objective function $F_{m}$ does not exceed $B$ if and only if copy $(i, j)$ is sequenced within the sequencing time window

$$
T_{(i, j) m}, i=1, \ldots, n ; j=1, \ldots, d_{i} .
$$

Proof. If the level curve

$$
f^{m}{ }_{i j}=n D\left|j-k r_{i}\right|^{m}
$$

of the objective function $F_{m}$ of TPRVP does not exceed a suitably chosen bound $B>0$, by Theorem 1, copy $(i, j)$ is sequenced at a time unit $k \in T_{(i, j) m} \subseteq[1, D]$. 
Conversely, let copy $(i, j)$ be sequenced at a time unit

$$
k \in T_{(i, j) m} \subseteq[1, D] .
$$

i.e., inequalities

$$
E_{m}(i, j) \leq k \leq L_{m}(i, j)
$$

hold. Thus,

$$
\begin{aligned}
& \left\lceil\frac{j-\sqrt[m]{\frac{B}{n D}}}{r_{i}}\right\rceil \leq k \text { and } \\
& k \leq\left\lfloor\frac{j-1+\sqrt[m]{\frac{B}{N D}}}{r_{i}}+1\right\rfloor
\end{aligned}
$$

which yields the inequalities

$\frac{j-\sqrt[m]{\frac{B}{n D}}}{r_{i}} \leq k$ and $k \leq \frac{j-1+\sqrt[m]{\frac{B}{N D}}}{r_{i}}+1$.

Thus, we get $n D\left|j-k r_{i}\right|^{m} \leq B$ and

$$
n D\left|(k-1) r_{i}-(j-1)\right|^{m} \leq B .
$$

\section{CONCLUSION}

For the total product rate variation problem, several pseudo-polynomial exact solution algorithms and heuristics have been developed. The earliest and the latest sequencing times of a feasible solution for TPRVP are

$E_{m}(i, j)=\left\lceil\frac{j-\frac{m}{\frac{B}{n D}}}{r_{i}}\right\rceil$ and $L_{m}(i, j)=\left\lfloor\frac{j-1+\sqrt[m]{\frac{B}{N D}}}{r_{i}}+1\right\rfloor$

respectively. These sequencing times can be used to develop an $O(D \log D)$ exact solution procedure recently given by Khadka and Werner (2014) which improves the known exact algorithm by Kubiak from (1993) with a complexity of $O\left(D^{3}\right)$.

\section{REFERENCES}

Brauner, N. and Crama, Y. 2004. The maximum deviation just-in-time scheduling problem. Discrete Applied Mathematics 134: 25-50.
Dhamala, T.N. and Khadka, S.R. 2009. A review on sequencing approaches for mixed-model just-intime production system. Iranian Journal of Optimization 1(3):266-290.

Dhamala, T.N., Khadka S.R. and Lee M.H. 2010. A note on bottleneck product rate variation problem with square-deviation objective. International Journal of Operations Research 7(1): 1-10.

Khadka S. R. 2012. Mixed-model just-in-time sequencing problem. Lambert Academic Publishing (LAP), Germany.

Khadka S. R. 2014. Estimation of the Largest and the Smallest Function Values of a Feasible Solution for the Total Product Rate Variation Problem. Journal of Science and technology 19(1): 35-38.

Khadka, S.R. and Werner, F. 2014. An exact solution procedure for the total product rate variation problem. Submitted.

Kovalyov M, Kubiak W. and Yeomans J. 2001. A computational analysis of balanced JIT optimization algorithms. Information Processing and Operational Research 39(3) 299-316.

Kubiak, W. 1993. Minimizing variation of production rates in just-in-time systems: A survey. European Journal of Operational Research 66:259-271.

Kubiak, W. and Sethi, S. 1991.A note on level schedules for mixed-model assembly lines in justin-time production systems. Management Science 37(1):121-122.

Kubiak, W. and Sethi, S. 1994. Optimal just-in-time schedules for flexible transfer lines. The International Journal of Flexible Manufacturing Systems 6:137-154.

Miltenburg, J. 1989. Level schedules for mixed-model assembly lines in just-in-time production systems. Management Science

35(2): 192-20 